\title{
Nanoconfinement-enhanced conformational response of single DNA molecules to changes in ionic environment
}

Reisner, Walter; Beech, J. P.; Larsen, Niels Bent; Flyvbjerg, Henrik; Kristensen, Anders; Tegenfeldt, J. 0.

Published in:

Physical Review Letters

Link to article, DOI:

10.1103/PhysRevLett.99.058302

Publication date:

2007

Document Version

Publisher's PDF, also known as Version of record

Link back to DTU Orbit

Citation (APA):

Reisner, W., Beech, J. P., Larsen, N. B., Flyvbjerg, H., Kristensen, A., \& Tegenfeldt, J. O. (2007).

Nanoconfinement-enhanced conformational response of single DNA molecules to changes in ionic environment. Physical Review Letters, 99(5), 058302. https://doi.org/10.1103/PhysRevLett.99.058302

\section{General rights}

Copyright and moral rights for the publications made accessible in the public portal are retained by the authors and/or other copyright owners and it is a condition of accessing publications that users recognise and abide by the legal requirements associated with these rights.

- Users may download and print one copy of any publication from the public portal for the purpose of private study or research.

- You may not further distribute the material or use it for any profit-making activity or commercial gain

- You may freely distribute the URL identifying the publication in the public portal 


\title{
Nanoconfinement-Enhanced Conformational Response of Single DNA Molecules to Changes in Ionic Environment
}

\author{
Walter Reisner, ${ }^{1,2,3}$ Jason P. Beech, ${ }^{2}$ Niels B. Larsen, ${ }^{1}$ Henrik Flyvbjerg, ${ }^{1}$ Anders Kristensen, ${ }^{3}$ and Jonas O. Tegenfeldt ${ }^{2}$ \\ ${ }^{1}$ Danish Polymer Centre and Biosystems Department, Ris $\phi$ National Laboratory, Technical University of Denmark-DTU, \\ DK-4000 Roskilde, Denmark \\ ${ }^{2}$ Department of Physics, Divison of Solid State Physics, Lund University, Box 118, S-221 00, Sweden \\ ${ }^{3}$ MIC-Department of Micro and Nanotechnology, Technical University of Denmark, DK-2800 Lyngby, Denmark \\ (Received 2 March 2007; published 1 August 2007)
}

\begin{abstract}
We show that the ionic environment plays a critical role in determining the configurational properties of DNA confined in silica nanochannels. The extension of DNA in the nanochannels increases as the ionic strength is reduced, almost tripling over two decades in ionic strength for channels around $100 \times 100 \mathrm{~nm}$ in dimension. Surprisingly, we find that the variation of the persistence length alone with ionic strength is not enough to explain our results. The effect is due mainly to increasing self-avoidance created by the reduced screening of electrostatic interactions at low ionic strength. To quantify the increase in selfavoidance, we introduce a new parameter into the de Gennes theory: an effective DNA width that gives the increase in the excluded volume due to electrostatic repulsion.
\end{abstract}

PACS numbers: 82.39.Pj, 81.16.Nd, 82.35.Lr

Understanding the behavior of dsDNA in confined geometries at the single-molecule level is crucial to the development of bionanofluidic technology for chip-based analysis systems. When the device dimension falls below characteristic molecular scales (e.g. the radius of gyration, persistence length), qualitatively new physical regimes are reached in which statistical properties of the macromolecule deviate from their values in bulk and become functions of the degree of confinement [1]. The effect of confinement in nanochannels is particularly striking: the DNA is observed to stretch out along the nanochannel [26]. As the DNA extension in the nanochannel scales linearly with the number of base pairs [2], the stretching creates a one-to-one mapping between genomic order and measured position along the stretched molecule, allowing the site specific interaction of biological enzymes and fluorescent probes to be measured with high accuracy [7].

The confinement of DNA in nanochannels greatly magnifies the molecule's conformational response to changes in ionic environment. The extension of DNA along a nanochannel almost triples over two decades' variation in ionic strength (see Fig. 1 and note that the ionic strength is defined as $I=\frac{1}{2} \Sigma_{i} z_{i}^{2}\left[p_{i}\right]$ in terms of the valence $z_{i}$ and the concentration $\rho_{i}$ of the $i$ th ionic species). The extension variation we observe cannot be explained by the ionic strength dependence of the persistence length, in contrast to recent interpretation of investigations of DNA stretching in $1000 \times 100 \mathrm{~nm}$ slit-nanochannel structures $[3,8]$. Over the 4-200 mM range in ionic strength our experiments access, the persistence length variation is not large enough to explain the observed change in extension. An additional physical mechanism is needed.

Ionic strength variation affects the polymer configuration by modulating the range of electrostatic interactions between charges on the DNA phosphate backbone. Electrostatic interactions in electrolyte solution are screened over a characteristic scale known as the Debye length, defined via its inverse $\kappa^{2}=\frac{2000 N_{A} e^{2} I}{\epsilon_{o} \epsilon k_{B} T}(e$ is the electronic charge, $\epsilon$ the dielectric constant of water, $\epsilon_{o}$ the permittivity of free space, $N_{A}$ Avagadro's number, $k_{B}$ Boltzman's constant, and $T$ the temperature [9]). The polymer geometry results in two types of electrostatic interactions [10]. As reference [3] argues, there are local repulsive interactions between charges separated by less than a Debye length in contour that increase the persistence length $P$. There are, however, additional interactions between charges greatly separated in contour that create repulsion between backlooping segments (i.e., greater excluded volume), leading to an effective DNA width $w_{\text {eff }}$ larger than the intrinsic width. We find that in fact these latter interactions are the dominant mechanism determining the ionic strength variation of the extension over our ionic strength range.

Single-molecule elasticity studies [11] suggest that $P$ follows the Odijk-Skolnick-Fixman formula [10]:

$$
P=P_{0}+\frac{1}{4 \kappa^{2} l_{B}}=P_{0}+\frac{0.0324 \mathrm{M}}{I} \mathrm{~nm}
$$

with $P_{0}$ the high salt value of the persistence length $(=50 \mathrm{~nm}), l_{B}$ the Bjerrum length $(\approx 0.7 \mathrm{~nm}$ in aqueous solution at room temperature). Equation (1) predicts that $P$ remains roughly equal to $P_{0}$ until the ionic strength drops below $10 \mathrm{mM}$. Between $10 \mathrm{mM}$ and $1 \mathrm{mM}$, it can rise to around $80 \mathrm{~nm}$.

The effective width is often estimated as being approximately the Debye length $(1 / \kappa)$, but this estimate is in fact incorrect. It is possible to develop a theoretical model for the excluded volume between two charged cylinders of diameter $w$, analogous to the classic expression for neutral cylinders, but taking into account the true electrostatic rodrod interaction. Onsager was the first to do so [12], and the calculation was later refined by Stigter and Fixman $[13,14]$. The main idea is (1) to calculate an effective rod- 
(a)

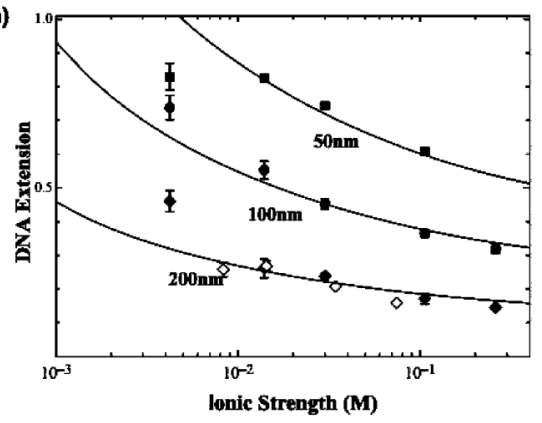

(b)

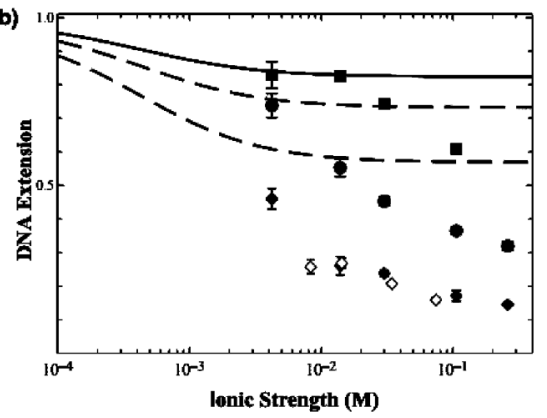

FIG. 1. Log-log plot of $\lambda$-DNA extension as a function of ionic strength for the three channel widths used (Filled diamonds, circles, and squares TBE series measurements in the 200, 100, and $50 \mathrm{~nm}$ channels respectively; open diamonds $\mathrm{NaCl}$ series measurements in the $200 \mathrm{~nm}$ channel). (a) Fits of the de Gennes scaling to extension measurements using the known ionic strength dependence of the effective width and persistence length. (b) The subpersistence length Odijk theory plotted against extension measurements (bold curve prediction for $50 \mathrm{~nm}$ channel, dashed curve prediction for $100 \mathrm{~nm}$ channel, and dot-dashed curve prediction for $200 \mathrm{~nm}$ channel). The de Gennes theory gives the better agreement for all three curves. The error bars given on the extension are the reported standard deviations from the extension of all molecules measured in the channel at a given ionic strength, between 5 and 20 molecules for the 100 and $200 \mathrm{~nm}$ channels and between 2 and 5 molecules for the $50 \mathrm{~nm}$ channel.

rod interaction potential using Poisson-Boltzmann theory, and then (2) use this interaction to evaluate the rod-rod excluded volume by evaluating the virial integral [10] over the rod positions and orientations. The result, for strongly charged chains, is

$$
w_{\text {eff }}=\frac{1}{\kappa}\left[0.7704+\log \left(\frac{\nu_{\text {line }}^{2}}{2 \epsilon \epsilon_{o} k_{B} T \kappa}\right)\right]
$$

with $\nu_{\text {line }}$ an effective DNA line charge discussed by Stigter in detail in reference [13]. Equation (2) suggests that $w_{\text {eff }}$ is indeed proportional to $1 / \kappa$, but with a prefactor that depends on the DNA charge and increases the estimate of $w_{\text {eff }}$. Techniques such as light scattering, sedimentation, and measurements of the probability of DNA knotting during cyclization [15] yields values for weff that are consistent and agree well with Eq. (2) (see reference [16] for an important plot that compares existing measurements of the effective width with the Stigter theory). Experiment and theory together suggest that the effective width de-

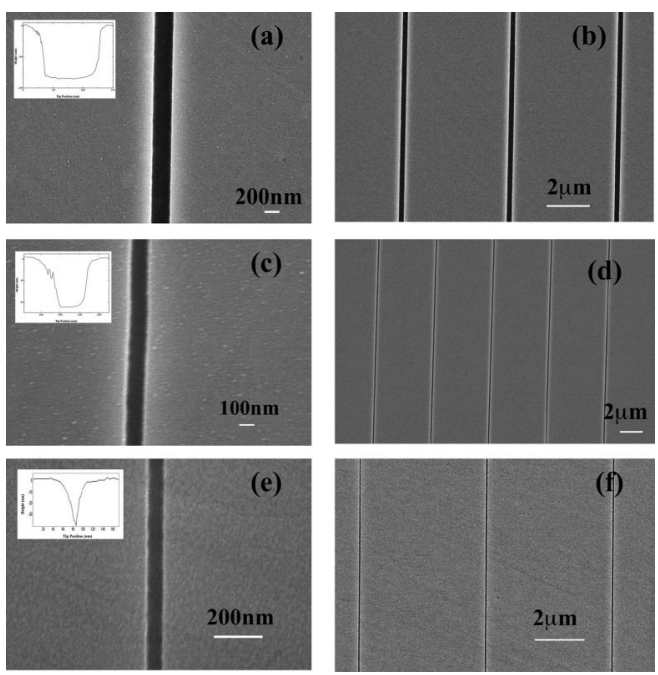

FIG. 2. SEM images of nanochannels. High magnification image of $200 \mathrm{~nm}$ (a), $100 \mathrm{~nm}$ (c), and $50 \mathrm{~nm}$ (e) channels with tapping mode AFM scan (inset). Low magnification SEM of $200 \mathrm{~nm}$ (b), $100 \mathrm{~nm}$ (d), and $50 \mathrm{~nm}$ (f) channels The channel dimensions can be estimated from these images with a $10 \%$ uncertainty.

pends strongly on the ionic strength, ranging from around $5 \mathrm{~nm}$ at $100 \mathrm{mM}$ salt to as high as $20 \mathrm{~nm}$ at $5 \mathrm{mM}$. Over the range in ionic strengths used in experiments, the variation of the effective width with ionic strength is much stronger than the persistence length variation.

When DNA is confined in channels with dimensions below the radius of gyration, the polymer conformation will be altered. Scaling arguments exist to explain the effect of nanochannel confinement on DNA conformation in the regimes where the channel width $D$ and height $h$ are either much greater or much less than $P$ [4]. In the regime $D, h \gg P$, the polymer is free to coil in the nanochannel, and de Gennes theory [17] predicts excluded volume interactions will create an extension $r$ along the channel linear with contour length $L$. The excluded volume $\chi$ between segments widely separated on the molecule contour can be modeled as the interaction between two hard rods of Kuhn length $2 P$ and width $w$ [4].

According to Onsager, $\chi \cong P^{2} w_{\text {eff }}[12]$. The Onsager excluded volume, combined with the de Gennes theory, leads to a relation for $r$ as a function of $D, h, P$, and $w_{\text {eff }}[4]$ :

$$
\frac{r}{L}=A\left(\frac{w_{\mathrm{eff}} P}{D h}\right)^{1 / 3} \text {. }
$$

The quantity $A$ is a geometry dependent numerical prefactor close to unity. Equation (3) shows that in the de Gennes regime, the factor $\left(P w_{\text {eff }}\right)^{1 / 3}$ describes the ionic strength dependence of the extension. Assuming that $P$ alone varies with ionic strength, Eq. (3) and Eq. (1) would predict only a $20 \%$ increase in extension over the entire ionic strength range. Yet, the large variation in $w_{\text {eff }}$ can create a significant additional variation in polymer extension explaining our measurements. 
Equation (3) is expected to break down as the channel dimensions fall below $P$. While the exact parameter regime where de Gennes theory should break down is unclear, there is evidence from nanoslit and nanochannel measurements that the de Gennes scaling holds approximately down to nanostructures at least $100 \mathrm{~nm}$ in dimension $[1,4]$. In the regime $D, h \ll P$, coiling is completely suppressed by the high bending energy [18] and contour can be stored within the polymer only through successive deflections of the polymer from the wall. By using T. Odijk's expression for the contour stored on average between polymer wall deflections [19], it is possible to show that $[3,4]$

$$
\frac{r}{L}=1-B\left[\left(\frac{D}{P}\right)^{2 / 3}+\left(\frac{h}{P}\right)^{2 / 3}\right]
$$

The quantity $B$ is a constant that theory suggests should have a value around 0.085 [3]. In contrast to the de Gennes theory, Eq. (4) suggests that in the subpersistence length regime the extension depends on the ionic strength through $P$ alone so that this theory is unlikely to explain the large observed variation in extension at high ionic strength.

The nanochannels used were fabricated in fused silica wafers (JINSOL). The channels were defined using electron beam lithography (JEOL) in zep520A resist [20] and then transferred to the underlying silica via $\mathrm{CF}_{4}: \mathrm{CHF}_{3}$ reactive ion etching (RIE). In order to explore the polymer physics in channels above and below the persistence length, channels were fabricated (see Fig. 2) with dimensions of roughly $50 \mathrm{~nm}\left(62 \times 44 \mathrm{~nm}^{2}\right)$, roughly $100 \mathrm{~nm}$ $\left(107 \times 91 \mathrm{~nm}^{2}\right)$, and roughly $200 \mathrm{~nm}\left(232 \times 173 \mathrm{~nm}^{2}\right)$. Further details regarding chip design can be found in $[2,4]$.

Experiments were conducted with $\lambda$-phage DNA (48.5 kbp, $L=16.5 \mu \mathrm{m}$, New England Biolabs). The DNA was dyed with YOYO-1 fluorescent dye (Molecular Probes) at a concentration of 1 dye molecule per every 5 base pairs. Staining the DNA increases the contour length. Fully stained $\lambda$-DNA has a contour length of $22 \mu \mathrm{m}$ at full intercalative loading of 1 dye/4 bp [21]. Assuming that the increase in contour length is linear with dye concentration, we estimate a contour length of $21 \mu \mathrm{m}$. We estimate the persistence length should increase by the same factor as $L$, so $P=64 \mathrm{~nm}$ at high ionic strength, agreeing within 5\% to the YOYO adjusted value of $P$ measured in [22].

The sealed devices were wet with dilutions of $0.45 \mathrm{M}$ tris-base $1 \mathrm{mM}$ EDTA with $0.45 \mathrm{M}$ boric acid $(5 \times \mathrm{TBE})$ at $p \mathrm{H}$ 8.5. TBE is a partially ionized system. In order to calculate the ionic strength from the buffer constituents, we have used the $p \mathrm{~K}_{\mathrm{a}}$ of Tris (8.1), boric acid (9.24), and EDTA $(1.99,2.67,6.16,10.26)$ and solved the full system of chemical equilibria [23] to obtain the concentrations of dissociated species $\left[\mathrm{TrisH}^{+}\right],\left[\right.$Borate $\left.^{-}\right]$, and $\left[\mathrm{EDTA}^{-3}\right]$ (EDTA is predominantly trivalent at the $p \mathrm{H}$ used). Some $\left[\mathrm{Na}^{+}\right]$is also included arising from titration and the disodium EDTA. The Davies equation was used to obtain the ionic strength dependence of the $p \mathrm{~K}_{\mathrm{a}}$ values [24]. Experiments with $0.05 \times \mathrm{TBE}$ plus $4 \mathrm{mM}, 10 \mathrm{mM}$,
$30 \mathrm{mM}$, and $70 \mathrm{mM} \mathrm{NaCl}$ were also carried out in the $200 \mathrm{~nm}$ channel and compare well to the TBE measurements. In addition, we used an anti-hotobleaching system consisting of $3 \% \quad \beta$-mercaptoethanol, $4 \mathrm{mg} / \mathrm{ml} \quad \beta$-Dglucose, $0.2 \mathrm{mg} / \mathrm{ml}$ glucose oxidase, and $0.04 \mathrm{mg} / \mathrm{ml}$ catalase. Note that $\beta$-mercaptoethanol is a weak acid $p \mathrm{~K}_{\mathrm{a}}=$ 9.6 [25], and we have included its effect on the chemical equilibria.

Using either electrophoresis or over-pressure, the DNA molecules were driven into the nanochannels from microchannel loading reservoirs. The extended DNA in the nanochannels at different TBE strengths were recorded with an iXon EMC CD camera (Andor) on a Nikon Eclipse TE2000 microscope using a $100 \times$ N.A. 1.4 oil immersion objective (Nikon) (see Fig. 3). The extension was calculated using the fitting function in [2]. Figure 1 presents the measured DNA extension as a function of ionic strength for the three channel widths used and compares the results to both Eq. (3) (Panel a) and Eq. (4) (Panel b). Equation (3) was fit to the data with $w_{\text {eff }}$ and $P$ determined, respectively, via Eqs. (2) and (1) using $A$ as a fitting parameter. The fit captures the ionic strength dependence of the extension down to $0.2 \times \mathrm{TBE}$ yielding values of $A$ close to unity. This agreement strongly suggests that the ionic strength dependence of the DNA extension can be described by the de Gennes model with electrostatically enhanced self-exclusion. On the other hand, Eq. (4) fails to agree with the measurements made in all three channels, both overestimating the extension and predicting too weak a variation with ionic strength. While it is not surprising that Eq. (4) fails to describe the 100 and $200 \mathrm{~nm}$ measurements (for which $D, h>P$, it is surprising that it does not

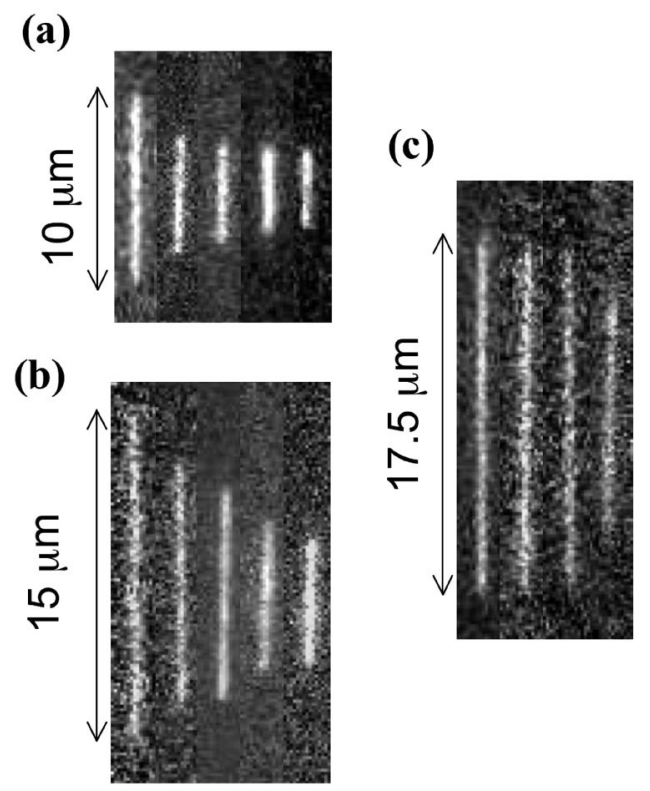

FIG. 3. A montage of individual fluorescently stained $\lambda$-DNA molecules measured at differing TBE dilutions (left to right, $0.05 \times, 0.2 \times, 0.5 \times, 2 \times, 5 \times \mathrm{TBE}$ ) in (a) $200 \mathrm{~nm}$ channels, (b) $100 \mathrm{~nm}$ channels, and (c) $50 \mathrm{~nm}$ channels. 
agree with the true subpersistence length measurements in the $50 \mathrm{~nm}$ channel $(D, h<P)$. Measurements at lower ionic strength might push our experiments clearly into a regime described by Eq. (4). Possibly, for the $50 \mathrm{~nm}$ channel, the threshold between the Odijk and de Gennes regimes lies between the $0.25 \times$ and $0.05 \times$ points at which the de Gennes scaling exceeds the measured extension values.

The de Gennes scaling underestimates the extension at $0.05 \times$ TBE for the 100 and $200 \mathrm{~nm}$ channels. This deviation is likely due to the existence of additional repulsive DNA channel wall interactions that become significant at low ionic strength. These interactions might take the form of entropic depletion interactions, as suggested by T. Odijk [18], additional electrostatic interactions, or a combination of the two. The complexity of these effects are such that the best theoretical approach is a full scale Monte Carlo simulation of a semiflexible, self-avoiding polymer with $w_{\text {eff }}$ determined by Eq. (2), using a realistic value for the zeta potential of the channel walls.

Jo et al. in [3] argue that Eq. (4) describes the extension of DNA in their slit anochannel devices over a range in ionic strength from 10 to $0.1 \mathrm{mM}$. It is not obvious that Eq. (4) is the correct scaling for their $1000 \times 100 \mathrm{~nm}$ channels, a hybrid situation where $h<P$ but $D \gg P$ so that coiling and back-bending are possible in the width dimension. This may be the factor leading to the saturation of their DNA extension at around 50\%.

This work demonstrates that the de Gennes scaling with electrostatically enhanced self-avoidance can explain the large stretching variation of nanochannel confined DNA with ionic strength in the $10-200 \mathrm{mM}$ range. From an applications point of view, nanochannels - as opposed to nanoslits - lead to an appreciable DNA extension over the more biologically relevant $10-100 \mathrm{mM}$ range where enzymes are fully functional and DNA is stable. Moreover, the sensitivity of the nanochannel-extended DNA conformation to the ionic environment suggests that the channels may become an important tool for further biophysical and biochemical investigations of DNA. In particular, we hope to study the effect of adding multivalent cations. Multivalent cations are known to decrease the effective width in manner not currently well understood [26], leading to the phenomena of DNA condensation [23,27].

This work was supported by the Danish Research Council for Technology and Production Science through Grant No. 274-05-0375 and the Swedish Research Council through Grant No. 2002-5972. We gratefully acknowledge Peixiong Shi for assistance with $e$-beam lithography at the Danchip clean room facility, Professor Austin of Princeton for helpful comments on draft versions, and Dr. Hsieh and Professor Doyle of MIT for helpful correspondence.

[1] D. Stein, F. H. J. Heyden, W. Koopmans, and C. Dekker, Proc. Natl. Acad. Sci. U.S.A. 103, 15853 (2006); A.
Balducci, P. Mao, J. Han, and P. Doyle, Macromolecules 39, 6273 (2006).

[2] J. O. Tegenfeldt, C. Prinz, H. Cao, S. Chou, W. Reisner, R. Riehn, Y. M. Wang, E. C. Cox, J. C. Sturm, P. Silberzan., and R. H. Austin, Proc. Natl. Acad. Sci. U.S.A. 101, 10979 (2004).

[3] K. Jo, D. Dhingra, T. Odijk, J. de Pablo, M. Graham, R. Runnheim, D. Forrest, and D. Schwartz, Proc. Natl. Acad. Sci. U.S.A. 104, 2673 (2007).

[4] W. Reisner, K. J. Morton, R. Riehn, Y. M. Wang, Z. Yu., M. Rosen, J. C. Sturm, S. Y. Chu, E. Frey, and R. H. Austin, Phys. Rev. Lett. 94, 196101 (2005).

[5] C.H. Reccius, J.T. Mannion, J.D. Cross, and H.G. Craighead, Phys. Rev. Lett. 95, 268101 (2005).

[6] L. J. Guo, X. Cheng, and C. F. Chou, Nano Lett. 4, 69 (2004).

[7] R. Riehn, M. Lu, Y. M. Wang, S. F. Lim, E. C. Cox, and R. H. Austin, Proc. Natl. Acad. Sci. U.S.A. 102, 10012 (2005); Y. M. Wang, J. O. Tegenfeldt, W. Reisner, R. Riehn, X.J. Guan, L. Guo, I. Golding, E. C. Cox, J. Sturm, and R.H. Austin, Proc. Natl. Acad. Sci. U.S.A. 102, 9796 (2005).

[8] M. Krishnan, I. Monch, and P. Schwille, Nano Lett. 7, 1270 (2007).

[9] J. Israelachvili, Intermolecular and Surface Forces (Academic Press, New York, NY, 1992).

[10] A. Grosberg and A. Khokhlov, Statistical Physics of Macromolecules (AmericanInstitute of Physics, NY, 1994).

[11] C. Baumann, S. Smith, V. Bloomfield, and C. Bustamante, Proc. Natl. Acad. Sci. U.S.A. 94, 6185 (1997).

[12] L. Onsager, Ann. N.Y. Acad. Sci. 51, 627 (1949).

[13] D. Stigter, Biopolymers 16, 1435 (1977).

[14] M. Fixman and J. Skolnick, Macromolecules 11, 863 (1978).

[15] T. Nicolai and M. Mandel, Macromolecules 22, 438 (1989); A. A. Brian, H. L. Frisch, and L. S. Lerman, Biopolymers 20, 1305 (1981); V. Rybenkov, N. Cozzarelli, and A. Vologodskii Proc. Natl. Acad. Sci. U.S.A. 90, 5307 (1993).

[16] A. Vologodskii and N. Cozzarelli, Biopolymers 35, 289 (1995).

[17] P. G. deGennes, Scaling Concepts in Polymer Physics (Cornell University Press, Ithaca, NY, 1979).

[18] T. Odijk, J. Chem. Phys. 125, 204904 (2006).

[19] T. Odijk, Macromolecules 16, 1340 (1983).

[20] SPIE Handbook of Microlithography, Micromachining and Microfab-Rication, edited by P. Rai-Choudhury (SPIE, Bellingham, WA, 1997), Vol. 1 Section 2.7.2.4.

[21] T. Perkins D. Smith, R. Larson, and S. Chu, Science 268, 83 (1995).

[22] S. Quake, H. Babcock, and S. Chu, Nature (London) 388, 151 (1997).

[23] A. Li, L. Qi, H. Shih, and K. Marx, Biopolymers 38, 367 (1996).

[24] D. Perrin and B. Dempsey, Buffers for $\mathrm{pH}$ and Metal Ion Control (Chapman and Hall, London, 1974).

[25] J. P. Danehy and C. J. Noel, J. Am. Chem. Soc. 82, 2511 (1960).

[26] V. Rybenkov, A. Vologodskii, and N. Cozzarelli, Nucleic Acids Res. 25, 1412 (1997).

[27] V. Bloomfield, Biopolymers 31, 1471 (1991). 